\title{
L'ANTHROPOLOGIE ENGAGÉE DANS LA LUTTE CONTRE EBOLA (2014-2016) : APPROCHES, CONTRIBUTIONS ET NOUVELLES QUESTIONS
}

\author{
Alice Desclaux, Julienne Anoko
}

S.F.S.P.| « Santé Publique »

2017/4 Vol. 29 | pages 477 à 485

ISSN 0995-3914

Article disponible en ligne à l'adresse :

https://www.cairn.info/revue-sante-publique-2017-4-page-477.htm

Distribution électronique Cairn.info pour S.F.S.P..

(C) S.F.S.P.. Tous droits réservés pour tous pays.

La reproduction ou représentation de cet article, notamment par photocopie, n'est autorisée que dans les limites des conditions générales d'utilisation du site ou, le cas échéant, des conditions générales de la licence souscrite par votre établissement. Toute autre reproduction ou représentation, en tout ou partie, sous quelque forme et de quelque manière que ce soit, est interdite sauf accord préalable et écrit de l'éditeur, en dehors des cas prévus par la législation en vigueur en France. Il est précisé que son stockage dans une base de données est également interdit. 


\title{
DOSSIER
}

Requestionner les approches de santé publique après Ebola

\section{L'anthropologie engagée dans la lutte contre Ebola (2014-2016) : approches, contributions et nouvelles questions}

\author{
Anthropology engaged against Ebola (2014-2016): \\ approaches, contributions and new questions
}

Alice Desclaux ${ }^{1}$, Julienne Anoko ${ }^{2}$, le Réseau ouest africain Anthropologie des Épidémies Émergentes (RAEE) impliqués dans le projet PACE (Programme d'Anthropologie Comparée d'Ebola)

\section{Ł Résumé}

Durant l'épidémie d'Ebola de 2014-2016, les anthropologues se sont engagés dans la riposte de trois manières : en tant qu'experts d'Ebola, médiateurs culturels entre populations et soignants, et chercheurs. L'article propose une esquisse de bilan de leurs approches et contributions, et discute les enjeux de cet engagement sur la base d'une revue de la littérature, d'études de cas et de débats lors de colloques. Les résultats de recherches en anthropologie présentés dans l'article concernent quatre thèmes : les contextes épidémiologiques de la transmission ; l'interprétation culturelle de la maladie et les réponses sociales ; la construction sociale de l'expérience des acteurs ; l'analyse critique des interventions de santé publique. Outre des contributions éclairantes en particulier sur les contextes sociopolitiques et leurs interfaces avec les mesures de santé publique globales, les anthropologues ont expérimenté des formes de communication de leurs résultats accessibles pour les acteurs de santé publique. Cependant, ces formes d'engagement hétérogènes soulèvent quelques questions, notamment lorsqu'elles reflètent des interprétations de l'anthropologie par ceux qui la sollicitent ou qui s'en revendiquent lui déniant toute portée critique ou réflexive, ou lorsqu'elles la confondent avec une intervention sociale. Néanmoins, les acquis de la recherche anthropologique sont importants, et pourraient l'être davantage si les réseaux transnationaux mis en place par les chercheurs pour analyser les dimensions sociopolitiques, économiques et bio-culturelles des épidémies émergentes pouvaient être pérennisés pour « préparer » la riposte à de futures crises sanitaires.

Mots-clés: Afrique de l'Ouest; Ebola; Anthropologie; Épidémie; Sciences sociales.

\section{$\lessdot$ Abstract}

Anthropologists contributed to the response to the 2014-2016 Ebola outbreak in three ways: as Ebola experts, cultural mediators between populations and caregivers, and researchers. This article presents a preliminary review of approaches, contributions and related issues based on a literature review, case studies and debates. The anthropological research discussed in this article concerns four themes: epidemiological contexts of transmission; cultural interpretation of illness and social responses; social construction of stakeholders' experience; critical analysis of public health interventions. In addition to insightful contributions, particularly regarding the socio-political contexts and their interfaces with global public health measures, anthropologists tested forms of communication to facilitate access of public health actors to their results. However, these heterogeneous forms of engagement raise a number of questions, especially when they reflect anthropological interpretations that exclude any critical or reflexive dimension, or when anthropology is considered to be similar to social intervention. Nevertheless, anthropological research provides a major contribution, which could be even greater if transnational networks set up by researchers to analyse the socio-political, economic and biocultural dimensions of emerging epidemics are supported in order to improve "preparedness" for future health crises.

Keywords: West Africa; Ebola; Anthropology; Epidemic; Social sciences.

\footnotetext{
1 TransVIHMI (UMI 233 IRD, U 1175 INSERM, Université de Montpellier) - IRD - 911 avenue Agropolis - F-34394 Montpellier - France.

${ }^{2}$ Anthropologue indépendante - Dakar - Sénégal.

${ }^{3}$ Les auteurs, chercheurs au Réseau ouest africain Anthropologie des Épidémies Émergentes sont les suivants : Francis Akindès, Blandine Bila, Moustapha Diop, Marc Egrot, Roch Houngnihin, Firmin Kra, Jeanne Madindé, Thierno Youla Sylla, Khoudia Sow, Bernard Taverne. CRCF Dakar Sénégal.
} 


\section{Introduction}

Lorsque l'épidémie ouest-africaine d'Ebola a été déclarée en mars 2014, l'Organisation Mondiale de la Santé (OMS) a affirmé rapidement la nécessité d'associer des anthropologues à la réponse sanitaire, suivie en cela par les principales organisations de santé globale multinationales ou non gouvernementales. Contrairement aux usages pour d'autres pathologies, l'OMS inclut systématiquement des anthropologues dans ses «équipes de réponse initiale » aux épidémies de « fièvres hémorragiques » d'Afrique centrale depuis 2003, dans l'objectif de comprendre les populations et d'« humaniser » les interventions sanitaires en les adaptant aux socio-cultures locales [1]. En Guinée, en 2014, les « réticences» des populations face aux mesures de santé publique ont, de plus, conduit les autorités sanitaires nationales et internationales à solliciter d'autres socio-anthropologues. Par ailleurs, des chercheurs en sciences sociales se sont eux-mêmes saisis de questions de recherche dans ce contexte de crise sanitaire. Ces trois « vagues » de mobilisation (qui ne sont pas tout-à-fait superposables à des séquences temporelles) correspondent à trois approches de l'engagement des sciences sociales, qui combinent de diverses manières des objectifs de connaissance et/ou d'intervention.

Si l'appel initial à mobiliser l'anthropologie face à l'épidémie d'Ebola semble avoir été entendu, son engagement n'allait pas de soi, car cette discipline s'accommode mal des contraintes temporelles. Elle a ses exigences épistémologiques, accordant de l'importance notamment à la mise en œuvre d'une « juste distance » entre chercheur et participants à la recherche, qui requiert à la fois l'immersion du chercheur dans un milieu socioculturel, une distanciation méthodologique et un détour théorique difficiles à mettre en place dans l'urgence [2]. De plus, la sollicitation précoce d'anthropologues ravive des questions anciennes et longuement débattues concernant la possible instrumentalisation des sciences sociales par la santé publique, les divergences entre les questionnements de ces deux champs disciplinaires, ainsi que le sens, les objets et les formes de communication pertinents entre chercheurs et acteurs de santé publique. Le contexte de la crise sanitaire générée par l'épidémie d'Ebola a-t-il permis des avancées sur cette interface? Répondre à cette question exige d'examiner les approches adoptées par des anthropologues et leurs contributions pendant cette épidémie en s'interrogeant sur le sens que revêt cette discipline pour les acteurs qui la convoquent comme pour ceux qui s'en revendiquent.

Un bilan des contributions de l'anthropologie face à l'épidémie d'Ebola de 2014-2016 sera nécessaire pour en discuter les acquis et limites afin d'en tirer des perspectives d'un point de vue de santé publique et d'un point de vue de sciences sociales. Cet article en présente une esquisse, car d'une part, divers aspects de l'engagement d'anthropologues en 2014-2016 restent à analyser, ce qui requiert des investigations spécifiques, en cours ; d'autre part, des publications en sciences sociales sont encore attendues.

\section{Méthodes}

L'article vise à répondre à deux questions : (1) Quelles ont été les formes particulières d'engagement des anthropologues face à l'épidémie d'Ebola en Afrique de l'Ouest? (2) Quels domaines thématiques et objets ont été explorés et quelles analyses ont été présentées ? Les réponses permettront de discuter en conclusion les enjeux de l'engagement d'anthropologues face à Ebola, au-delà de la flambée épidémique 2014-2016.

Ces questions sont abordées sur la base: (1) des recherches menées par les auteurs de l'article qui ont fourni des études de cas (à partir notamment du Projet d'Anthropologie Comparée d'Ebola ${ }^{1}$ ) ; (2) d'une revue de la littérature incluant les périodiques scientifiques internationaux (en anthropologie et santé publique), les sites internet dédiés (notamment Ebola Response Anthropology Platform ${ }^{2}$ et le site du Réseau ouest-africain Sciences Humaines et Sociales Ebola $^{3}$ ) et la littérature grise parue sur des listes de diffusion spécialisées ${ }^{4}$; (3) de conférences tenues en Afrique de l'Ouest (notamment le colloque Ebodakar ${ }^{5}$ ). L'article porte particulièrement sur l'espace francophone ouest-africain incluant les pays touchés par l'épidémie ou en situation de risque épidémique.

\footnotetext{
${ }^{1}$ Projet qui rassemble des études au Sénégal, en Guinée, au Bénin, en Côte d'Ivoire et au Burkina Faso (coordination B. Taverne, financement IRD, Aviesan, Expertise France, Unicef)

${ }^{2}$ www.ebola-anthropology.net

${ }^{3}$ shsebola.hypotheses.org, RSHSE devenu RAEE en janvier 2016.

${ }^{4}$ Principalement trois listes animées par A. Epelboin, R. Migliani et S. Abramowitz, pendant la durée de la crise.

${ }^{5}$ Ce colloque, intitulé «Épidémie d'Ebola en Afrique de l’ouest. Études ethnographiques comparées » a rassemblé environ 150 spécialistes des sciences sociales et de la santé publique à Dakar du 19 au 21 mai 2015. Un bilan scientifique est disponible à l'adresse: http:// ebodakar2015.sciencesconf.org/resource/page/id/7
} 


\section{Des formes singulières d'engagement des anthropologues}

Les trois «vagues » d'engagement de l'anthropologie dans le contexte de l'épidémie d'Ebola (2014-2016) renvoient à deux modèles (les experts Ebola et les médiateurs culturels) coexistant avec des formes plus usuelles d'exercice de la recherche (comprises ici sous le libellé chercheurs en anthropologie). Ces modèles ne constituent pas une typologie des chercheurs, car certains d'entre eux ont adopté successivement plusieurs formes d'engagement.

\section{Les experts Ebola}

La première étude de terrain systématique en anthropologie sur Ebola, menée à la fin de l'épidémie de 2000-2001 en Ouganda, montrait la variété des interprétations (étiologie, symptômes, traitement de la maladie à virus Ebola -MVE) par les populations [3] ; ses auteurs concluaient sur la nécessité d'investiguer ces aspects majeurs pour l'acceptabilité des interventions. Confrontée aux « réticences » des populations au Gabon et au Congo pendant les épidémies de 2000 et 2001, l'OMS associe des anthropologues dans «l'équipe de réponse initiale » à l'épidémie de 2003 (au Congo), puis lors des épidémies ultérieures, constituant un pool d'experts (notamment B. Hewlett, A. Epelboin, J. Anoko). Ces scientifiques académiques connaissent la maladie, ses aspects médico-techniques, les contraintes de biosécurité, l'histoire des réactions des populations lors d'épidémies antérieures, les dimensions institutionnelles (Règlement Sanitaire International, rôle de l'OMS et des institutions sanitaires globales et locales) ; ils sont reconnus dans des réseaux sociaux spécialisés et communiquent dans les institutions de santé globale. Parmi eux, B. Hewlett publie la première analyse approfondie des aspects imaginaires et symboliques des épidémies successives de fièvre hémorragique au plan local et au plan international [4]. A. Epelboin utilise les notions et l'expérience acquises par l'anthropologie médicale appliquée aux maladies infectieuses et pratique l'immersion « symétrique » auprès de la population et parmi les acteurs de la riposte ; grâce à son double statut d'anthropologue et médecin ainsi qu'à son insertion dans l'équipe de l'OMS, il produit de manière corrélée à ses enquêtes des recommandations destinées aux professionnels de santé qui peuvent ainsi adapter leurs techniques. De plus, il prend le parti de produire des films documentaires ayant un objectif pédagogique et éthique (en application du principe de transparence) [5], qui montrent les procédures de prise en charge, les pratiques de biosécurité en contexte communautaire et les réactions des populations ${ }^{6}$. Lorsque l'épidémie est déclarée en Guinée, l'OMS fait immédiatement appel à ces experts pour mettre en œuvre «l'humanisation » des mesures de santé publique, expliciter les interprétations de la maladie par les populations ainsi que les logiques sociales sous-jacentes à leurs réactions. Les productions d'anthropologues experts tels que le film d'A. Epelboin (encadré 1) ont une composante essentielle de documentation, due à leur présence sur le terrain dès les débuts de l'épidémie, et contribuent à la constitution d'archives des dimensions sociales des flambées épidémiques et de leur gestion sanitaire et sociopolitique.

Encadré 1 : Le film " Ebola en Guinée Conakry : Guéckédou, hôpital sécurisé par MSF, avril 2014 " [7]

Ce film présente le premier Centre de Traitement Ebola (CTE) de Guinée et les tensions sociales qui s'y jouent. Son intérêt est d'abord pédagogique : il permet à des professionnels de santé de comprendre et discuter les difficultés quotidiennes de la prise en charge qu'ils ne connaissent encore qu'au travers des recommandations et manuels, levant une inquiétude face à l'inconnu. De plus, les éléments documentaires concernant les populations comparés à ceux produits lors d'épidémies antérieures, constituent une base de connaissances empiriques qui montre la récurrence des situations "à risque social " et les stratégies utilisées, qu'elles aient réussi ou échoué. Son intérêt est donc triple : didactique pour les professionnels de santé, offrant des observations en situation pour discuter les pratiques de soins et les aspects éthiques, et documentaire pour les anthropologues.

\section{Les médiateurs culturels}

En Guinée, les premières réactions de refus des soins s'expriment rapidement mi-2014 et les familles commencent à cacher les malades, d'autant plus que certaines autorités nationales critiquent les intervenants internationaux de la riposte. La gravité de la maladie et les interventions sanitaires spectaculaires, intrusives et parfois violentes, suscitent la peur, tels les soignants masqués dans leurs équipements de protection, les désinfections autoritaires des espaces domestiques, l'évacuation forcée des malades qu'on ne reverra plus vivants, la destruction de leurs objets personnels, les corps placés dans des sacs mortuaires sans que puissent être réalisés les rituels funéraires. Les interprétations persécutives sont favorisées par ce climat de peur éprouvée par les acteurs de santé comme par les populations, et alimentent les rumeurs. De plus, les premiers messages de santé publique diffusés dans la sous-région, négligeant ou ignorant l'expérience acquise à propos de l'infection à VIH, favorisent la stigmatisation : ils soulignent le

\footnotetext{
${ }^{6}$ Voir notamment « Ebola, ce n'est pas une maladie pour rire » [6].
} 
risque que représentent les malades sans expliciter les soins qui leur seront apportés ni s'adresser directement à eux. Cette «épidémie de peur » qui précède et accompagne la diffusion du virus est modulée par les rapports pré-existants aux systèmes de soins et aux autorités sanitaires. Dès juin 2014, des scientifiques soulignent la nécessité du recours à la médiation sociale pour instaurer rapidement la confiance nécessaire au contrôle de l'épidémie dans les trois pays touchés [8]. Des anthropologues sont sollicités en premier lieu par l'OMS et l'Unicef avec pour missions de comprendre les perceptions et attitudes des populations, d'identifier les leaders d'opinion et des « alliés » pour la communication, de suggérer des messages adaptés, et de soutenir la constitution de comités locaux de lutte contre Ebola dans le but de faciliter le déploiement des interventions sanitaires et de favoriser la participation communautaire à la riposte [9]. Le rôle attribué à ces anthropologues est diversement interprété : certains agissent en porte-paroles des « communautés » ou en « médiateurs culturels » [10], d'autres révèlent les besoins fondamentaux des populations dont la prise en compte apparaît comme une condition pour la réduction de la méfiance vis-à-vis des autorités nationales [11]. Ce travail, spécifique des configurations microsociales à l'échelle locale (Encadré 2), exige un investissement rapide avant que les tensions sociales ne dégénèrent en conflits, important au vu du grand nombre de sites concernés. Il doit aussi être renouvelé dans le temps, car, comme l'indique C. Niang, après une intervention pour l'OMS en Guinée : «Quand on a fini dans un village, tout peut être à refaire dans le village voisin $»^{7}$.

Encadré 2 : L'anthropologue médiateur entre populations "résistantes " et acteurs sanitaires

Parmi les quelques expériences qui ont fait l'objet d'une publication, S. Faye décrit le conflit violent entre "cadets sociaux" (jeunes et femmes) et aînés du "conseil des sages" ayant des approches différentes de la protection contre l'épidémie dans le village de Kolobengou, l'un des 26 villages de Guinée où des "réticences" étaient identifiées en juillet 2014. C'est en engageant une stratégie basée sur l'écoute des acteurs sociaux, l'organisation d'espaces d'expression et d'échanges, et la définition commune d'actions collectives impliquant les jeunes contre Ebola, que la situation a pu être pacifiée et qu'il a été possible de "faire émerger une solidarité communautaire positive" permettant la collaboration avec les acteurs sanitaires [12]. Ces interventions exigent de la part de l'anthropologue une bonne connaissance des logiques culturelles en Afrique de l'Ouest et à une échelle plus locale (pays, village, région), un travail minutieux et patient pour se faire accepter ainsi qu'une grande disponibilité vis-à-vis des particularités locales [10].

\footnotetext{
${ }^{7}$ Conférence sur les risques sanitaires, CODESRIA, Dakar, 13 août 2014.
}

\section{Les chercheurs en " anthropologie "}

L'épidémie d'Ebola a également fait l'objet d'études et recherches en sciences sociales proposées par des sociologues et anthropologues, à leur propre initiative ou sur la sollicitation d'institutions ou de projets de recherche clinique. Le qualificatif « anthropologique » a été appliqué à des travaux assez divers, pouvant inclure des études rapides focalisées autour de questions prédéfinies (connaissances et représentations de la maladie, attitudes, «croyances » sur l'origine), d'approches quanti- ou qualitatives, procédant par questionnaires sur la base d'échanges individuels ou collectifs ; à l'opposé, d'autres recherches, éclairées par un ancrage théorique et utilisant des méthodes ethnographiques rigoureuses, ont été menées sur des thématiques plus ajustées aux contextes. Le premier type d'études, sollicité par des institutions internationales, nécessitant une expertise limitée, est financé plus aisément et réalisé plus rapidement que le second; il a permis, au cours de l'épidémie, à des universitaires et à des consultants de découvrir les sciences sociales de la santé dans un contexte de prestations ne donnant lieu que rarement à des publications scientifiques. Le second type, qui relève de la recherche, nécessite un meilleur niveau méthodologique et une connaissance préalable des cultures et/ou des questions de santé en Afrique de l'Ouest ainsi que du vaste domaine conceptuel exploré par l'anthropologie médicale : anthropologie du corps, des maladies infectieuses, de la recherche clinique, de la santé globale, de la santé publique, etc. ${ }^{8}$. Ainsi, au cours de l'épidémie, l'approche anthropologique définie par les commanditaires a-t-elle dans certains cas été brouillée par l'exigence de résultats rapides sans considération pour les connaissances et les savoir-faire fondamentaux de cette discipline, indispensables pour produire des analyses scientifiquement pertinentes et définir des recommandations opérationnelles efficientes.

\section{Des objets de la recherche en contexte épidémique}

Dans les limites de cet article, la revue des objets de recherche aborde quatre thématiques majeures dans une perspective de contribution à la riposte, qui ne couvrent que partiellement l'ensemble des travaux de recherche réalisés en anthropologie.

\footnotetext{
${ }^{8}$ Les travaux réalisés dans cette approche sont présentés dans la section suivante.
} 


\section{Les contextes de la transmission}

L'étude de l'exposition au risque et des modalités de transmission est en premier lieu dévolue aux épidémiologistes, qui ont progressivement apporté des éléments de connaissance sur les chaines de transmission du virus Ebola, les facteurs de risque et profils des populations touchées, peu élucidés lors des épidémies précédentes [13]. Les travaux d'anthropologues éclairent les conditions de la transmission en contextualisant ces connaissances. Des études menées avant 2014 dans une perspective écologique avaient commencé à explorer les interactions entre hommes et espèces animales réservoirs en jeu dans les anthropozoonoses en Guinée [14]. Ce type d'étude a contribué en particulier à deux débats, toujours d'actualité, concernant d'une part les contacts avec des chauves-souris dans l'émergence de la flambée ouest-africaine et d'autre part l'importance de la viande de brousse dans la transmission, probablement surestimée; cette dernière question n'est pas anodine car l'interdiction de consommation adoptée trop rapidement et trop largement comme mesure préventive dans tous les pays à risque épidémique a généré des conflits sociaux, eu des conséquences socioéconomiques délétères, et contribué à des réactions de déni ou des interprétations en termes de complot. Un apport analytique majeur fut l'élargissement de l'approche naturaliste à une perspective bioculturelle qui a éclairé l'articulation des facteurs écologiques et biologiques et des facteurs économiques, sociaux et politiques, analysés par des anthropologues sociaux et des historiens [15] [16] [17], tels que « l'héritage colonial, l'ajustement structurel, l'exploitation par les compagnies minières, qui ont favorisé les guerres civiles, la pauvreté du monde rural et la quasiabsence de soins de qualité » [18]. Ces travaux portant sur les contextes et déterminants méso- et macro-sociaux de l'émergence de l'épidémie - ou «facteurs structurels » - ont une valeur analytique importante ${ }^{9}$, en particulier lorsqu'ils abordent les dimensions politiques de l'exposition au risque et les questions d'échelle (locale, régionale, nationale et globale). Dans une perspective de réponse, des études focalisées sur des pratiques et logiques sociales qui favorisent l'exposition au virus, concernant par exemple les rituels funéraires (Encadré 3), sont indispensables pour définir une prévention pertinente et adaptée.

\footnotetext{
${ }^{9}$ On accède à ces analyses en particulier sur le site Ebola Response Anthropology Platform.
}

Encadré 3 : Les rituels funéraires en Côte d'Ivoire (2015). F. Kra, M. Egrot, F. Akindès

Lors de l'épidémie, des injonctions interdisant les rituels funéraires ont été diffusées dans les pays touchés ou à risque épidémique, ces rituels étant considérés comme le mode principal de diffusion du virus lors des contacts avec le corps des personnes mourantes ou décédées de MVE. En Côte d'Ivoire, ils sont englobés dans une catégorie : "Pratiques traditionnelles dangereuses ".

Les données collectées dans le cadre du programme Ebo- $\mathrm{Cl}$ : "Côte d'Ivoire et MVE " à Abidjan, Noé et Tabou montrent que, malgré l'intensité des campagnes préventives, ces rituels restent inchangés. Un autre constat est leur diversité selon les aires culturelles, avec, au sein d'une même population, une variabilité et une spécificité en fonction : du statut social de la personne; du type de mort (mauvaise/ bonne, violente/attendue, "naturelle "/suspecte); des modèles étiologiques de l'évènement; du lieu du décès. Diversité, variabilité et spécificité imposent d'avoir une connaissance ethnographique des rituels avant d'en proposer des adaptations.

Ces rituels sont par ailleurs flexibles et malléables. Ils sont aussi associés à des risques perçus, en lien avec leur exécution, et des contre-rites proposés comme mesures prophylactiques en cas d'erreurs commises dans leur réalisation. Considérés comme l'ensemble des pratiques depuis le trépas jusqu'à l'ancestralisation, ces rituels permettent d'objectiver la mort, d'en trouver la cause, de réduire les rumeurs et les discours subjectifs construits sur des hypothèses étiologiques. Ils ont pour fonction de réduire le poids des responsabilités relatives à l'événement et d'estomper d'éventuelles culpabilités ressenties ou assénées. Ils constituent des mécanismes de résilience construits par les sociétés face à la mort. Impératifs culturels, en particulier religieux, ils apparaissent dans les imaginaires collectifs comme une "norme sociale", mais surtout comme une pratique permettant le maintien d'une sociabilité optimale entre les vivants et une reproduction sociale. Inscrits au cœur des savoirs collectifs, ils contribuent aussi à une restauration itérative de l'ordre social. Les risques potentiellement induits par leur transgression apparaissent aux populations comme bien plus importants que le risque, lointain et hypothétique, d'une éventuelle transmission de la MVE.

Même s'ils apparaissent difficiles à articuler avec des mesures de biosécurité, une meilleure connaissance de ces rituels permettrait de construire des procédures circonstancielles adaptées, utilisant le caractère malléable des pratiques, la perception d'autres types de risques induits par ces rituels, ou encore les contre-rites déjà existants localement.

\section{L'interprétation culturelle de la maladie et les réponses sociales}

Avant même l'apparition de cas localement, les réactions des populations aux informations sur la brutalité et la gravité de la maladie diffusées à propos de la MVE étaient marquées par la peur et le rejet, et influencées par une communication utilisant cette peur dans un but pédagogique. Les rumeurs, souvent relayées et légitimées par les médias, ont donné leurs formes aux représentations profanes de la maladie, surestimant le risque de transmission, mettant en doute les mesures et intentions des 
intervenants sanitaires (notamment la désinfection des domiciles et les Centres de Traitement Ebola -CTE) et «accusant les victimes» [11]. Les tensions, qualifiées de « réticences » par l'OMS, ont été paroxystiques en Guinée, allant de l'absence de confiance envers des autorités sanitaires qui blâmaient les populations pour leur « ignorance » jusqu'au meurtre d'acteurs de prévention [19]. Plus globalement, les réponses sociales à l'épidémie en termes d'interprétations et d'actes ont été hétérogènes : instauration de protections physiques (mise à distance des malades ou catégories sociales et identitaires perçues comme associées à la maladie), observance de mesures de biosécurité ayant une dimension symbolique et sociale (lavage des mains, prise de la température), mobilisation d'acteurs sociaux en conjonction ou parallèlement aux mesures de santé publique (implication des religieux et de divers « groupes

Encadré 4 : Les logiques sociales de la destruction d'un centre de traitement d'Ebola au Bénin (2015). M. Egrot, R. Houngnihin, J. Madindé

En 2015, un centre de traitement d'Ebola (CTE) construit par le Ministère de la santé du Bénin pour accueillir d'éventuels cas de MVE est détruit par la population la veille de son inauguration. Le programme EbBen: "Anthropologie d'Ebola au Bénin" s'est fixé comme objectif de comprendre les logiques expliquant cet acte de violence. Une approche qualitative a permis de collecter des données sur l'histoire de ce centre et sur les évènements.

Fin août, le Ministère de la santé choisit de construire un CTE dans un quartier populaire excentré, au Nord de Cotonou, sur un terrain jouxtant le centre de santé, initialement destiné à une association de tradipraticiens. Le Comité de gestion du centre de santé (COGECS) adresse un courrier de contestation au chef d'arrondissement. Faute de réponse, il envoie une motion de protestation à diverses autorités sanitaires, administratives et politiques, locales et nationales. Puis une association d'autochtones du quartier envoie à la Ministre de la santé un courrier de dénonciation, principalement sur les risques de "contagion" et la proximité du collège. Durant cette phase de protestations, les travaux continuent. Deux séances d'information ont lieu en octobre entre les autorités sanitaires et le COGECS. L'objectif est d'organiser une sensibilisation des populations qui n'aura jamais lieu faute de moyens. Le 31 octobre 2015, une partie de la population se soulève et empêche l'accès au site. Une séance d'information a lieu immédiatement avec quelques notables et leaders religieux: un accord est trouvé pour continuer les travaux. Dans la nuit suivant son achèvement, le CTE est saccagé, avant l'inauguration par le Chef de l'État, la veille de la 64ème session du comité régional de l'OMSAfrique à Cotonou.

L'analyse des évènements montre une diversité d'acteurs sociaux mobilisés autour du CTE, avec des logiques d'actions et des intérêts très différents. L'autre fait marquant est la construction sociale très rapide de la polémique, des rumeurs et enfin de la violence. Précipitation, insuffisance de la communication, contradictions, maladresses des autorités, usages politiques sont autant d'explications possibles, qui, combinées avec le contexte de peur de la contagion, ont abouti à une altération de la confiance, une montée de la colère, et enfin une émergence de la violence. communautaires »). Ces réactions contrastées résultent, pour ce qui concerne la Guinée, d'une histoire de dissensions, méfiance et violence dans le rapport entre État et populations, et reflètent la politisation de la santé publique [20]. Dépendantes des contextes sociopolitiques locaux (cf. Encadré 4), ces réactions ont aussi évolué en suivant la riposte, les survivants d'Ebola ayant un discours positif sur leur expérience en CTE, ce qui montre que les interventions de « santé globale » modifient non seulement les paysages thérapeutiques mais aussi les perceptions des systèmes de soins, en fonction de leur organisation sociopolitique [21].

\section{La construction sociale de l'expérience des acteurs}

L'un des rôles-clés de l'anthropologue est d'explorer l'expérience et le point de vue d'acteurs sociaux, dans leur propre logique, pour documenter des pans de l'expérience humaine, et " porter la voix » de ceux dont le statut social ne leur permet pas d'être entendus. Comme pour l'épidémie de sida, les personnes atteintes de MVE ont été stigmatisées (discriminées, dénuées de reconnaissance, culpabilisées et socialement exclues), un phénomène qui a persisté après que les craintes de contagion se soient estompées. L'exploration de cette expérience a permis à des anthropologues de proposer des outils pour limiter l'impact de stratégies de lutte contre Ebola en termes de stigmatisation [22]. Ailleurs, des approches graduées et des rituels de réintégration sociale ont été mis en œuvre ; l'analyse visait à comprendre leur impact sur la volonté des survivants et de leurs communautés de reconstruire la cohésion sociale [23]. Au-delà de l'exclusion, l'analyse des déterminants sociaux de l'expérience (cf. Encadré 5) est importante pour les intervenants socio-sanitaires car elle permet de comprendre si les soins et aides apportés correspondent aux attentes des personnes dans leur contexte socioculturel spécifique et dans leur diversité. L'épidémie d'Ebola a généré d'autres formes d'expérience singulières, en cours d'étude, telles que celle des volontaires engagés dans la gestion des enterrements sécurisés, et celle des soignants qui ont été eux-mêmes atteints et rencontrent désormais les difficultés des survivants [24].

\section{L'analyse critique des interventions de santé publique}

A. Kleinman, autorité scientifique en anthropologie médicale, considère que l'analyse critique des politiques et pratiques de la santé publique globale constitue la contribution majeure de ce champ disciplinaire [25]. En 2014, les stratégies et interventions de santé publique ont d'abord 
Encadré 5 : Le vécu des femmes survivantes d'Ebola (2016). B. Taverne, T. Y. Sylla

Le vécu des femmes guéries de la MVE a été étudié dans le cadre du programme de recherche interventionnelle et multidisciplinaire Postebogui (Évaluation et accompagne-ment des patients déclarés guéris d'une infection par le virus Ebola en Guinée). L'analyse aborde le vécu de la maladie et de la guérison, les formes de désocialisation et resocialisation, la stigmatisation et les conséquences psychosociales individuelles et familiales, ainsi que les perceptions et attitudes vis-à-vis du système de soins et de la recherche parmi divers groupes sociaux de personnes guéries (femmes, hommes, adolescents et jeunes adultes, enfants, professionnels de santé).

Une première série d'entretiens montre l'importance de la MVE parmi les évènements signifiants de leur vie. Au moment de l'entretien, soit entre un et deux ans après la sortie du CTE, les situations sont très diverses : retour à une "vie normale " volontariste mais marqué par la mémoire de l'épreuve; quotidien dominé par les séquelles physiques de la maladie; déclassement social dû à la perte du conjoint; investissement notamment dans l'accueil d'enfants orphelins confiés; expérience d'engagement comme "patient expert", désormais sans activité. L'approche narrative basée sur des entretiens approfondis a permis de montrer le sens que les personnes donnent à leur atteinte, de décrire des processus psychosociaux d'imputation ou de culpabilisation post-traumatique ainsi que des besoins en termes d'aide sociale ou au plan psychique que l'approche médicale n'avait pas identifiés précisément.

été définies localement dans l'urgence, à partir de connaissances scientifiques limitées, selon une rationalité essentiellement sanitaire et logistique, et sous l'emprise de la peur qui n'a pas épargné les intervenants au vu de l'extension incontrôlée de l'épidémie [26]. Dans ce contexte, certaines mesures ont été inacceptables pour les populations, et de ce fait improductives d'un point de vue de santé publique : la gestion initiale des « enterrements sécurisés » ne laissant aucun rôle à l'entourage du défunt, entre autres exemples, a été dénoncée. L'organisation des interventions sanitaires a également produit des formes de souffrance, ce que montre F. Le Marcis dans son analyse du traitement des corps des malades décédés dans les CTE, dont l'absence d'identification après leur inhumation non seulement contrevenait à la dignité du défunt mais empêchait l'entourage d'accomplir des rituels de deuil et d'ancestralisation [27]. L'analyse critique des dispositifs de santé publique a aussi mis en évidence les conditions qui rendent des mesures sanitaires acceptables, par exemple dans le cas de la surveillance sanitaire des sujets contacts [28]. La comparaison entre sites (Encadré 6) montre qu'une mesure sanitaire peut être acceptable sous conditions dans un contexte particulier, mais se révéler délétère sur le plan social et sanitaire dans un autre contexte.
Encadré 6 : Les perceptions de la surveillance par les sujets contacts au Sénégal et en Guinée (2015). A. Desclaux, M. Diop

Les personnes qui ont été exposées au risque de MVE, identifiées comme contacts, doivent faire l'objet d'une surveillance sanitaire pendant 21 jours, où elles sont confinées au domicile. Au Sénégal, 43 des 74 personnes ayant été en contact avec le "cas importé d'Ebola " ont fait l'objet d'entretiens approfondis sur leur expérience, dans le cadre du Projet EBSEN : "Épidémie d'Ebola et production sociale de la confiance au Sénégal ". En Guinée, des observations et entretiens ont été réalisés dans le cadre d'une mission de terrain appuyée par I'Unicef dans la région de Kaliya.

L'analyse des perceptions des sujets contacts montre que la quarantaine, selon leurs termes, est vécue comme une mesure nécessaire, car ils adhèrent à la mise à distance comme mesure préventive et parce qu'elle permet de se protéger des réactions agressives des tiers. Cependant, l'observance du confinement qui suspend les activités professionnelles laisse sans ressources les personnes et leurs dépendants; de plus, l'isolement peut être difficile à supporter sur le plan psychologique, surtout si on est stigmatisé. L'appui alimentaire et financier a été instauré dans les deux pays de manière pragmatique, mais ces mesures doivent accompagner systématiquement la mise en quarantaine pour en garantir la faisabilité, et être gérées de manière plus équitable. Les observations montrent aussi que les pathologies intercurrentes et les troubles psychiques induits par la quarantaine doivent être soignés, ce qui requiert la mise en place d'un dispositif d'écoute et de soin à domicile. Enfin, au-delà de l'identification des mesures d'accompagnement nécessaires pour éviter que les mesures de quarantaine n'induisent une iatrogénie, l'analyse a montré que la pression sociale à la recherche des sujets contacts, généralisée à la fin de la flambée épidémique en Guinée, suscitant la crainte, pourrait être en cause dans la disparition de personne(s) identifiée(s) comme telle(s).

\section{Discussion}

L'engagement précoce des anthropologues sur sollicitation, parallèlement à la mise en œuvre de recherches à leur initiative, témoigne d'une forme de reconnaissance de l'importance du « social » en santé publique que les chercheurs en anthropologie de la santé réclament depuis plusieurs décennies. Ils ont investi l'épidémie d'Ebola de 2014-2016 en mobilisant des formes nouvelles d'engagement et en produisant des connaissances clés pour la compréhension de l'épidémie et la pertinence de la riposte, dont seulement quelques aspects ont été présentés dans cet article. Les anthropologues se sont aussi investis pour que leur discipline soit utilisée de manière pertinente, entre autres en commençant par publier un inventaire de «ce que les anthropologues peuvent faire pour lutter contre l'épidémie d'Ebola » à l'intention des acteurs de la riposte [29]. Pour la communication de leurs analyses ils ont utilisé, en complément aux publications scientifiques, des formes plus accessibles aux acteurs de santé : présentations 
synthétiques résumées (policy briefs) usuelles dans la culture des agences des Nations-Unies, films, communications brèves et rapides, sites internet dédiés, affiches et kakémonos, réseaux et rencontres inter-disciplinaires.

Cependant, cet engagement ne fut pas sans écueils. L'intérêt affiché pour l'anthropologie ne s'est accompagné qu'exceptionnellement d'une mobilisation de ressources financières à hauteur des sollicitations; lorsque ces ressources ont été obtenues, ce fut souvent trop tardif, avec des durées de contrats trop limitées. Au vu des budgets investis, «le social» (incluant les dimensions sociales, culturelles, historiques, économiques, politiques) est resté le «parent pauvre » de la recherche en santé sur l'épidémie d'Ebola. De plus, une méconnaissance des sciences sociales assez répandue dans les institutions médicales ainsi que l'absence de spécialisation des savoirs professionnels de l'action sociale dans les pays touchés ont conduit à confondre dans les sollicitations des problématiques relevant de disciplines diverses (psychologie, travail social, psychiatrie, économie, communication) pour lesquelles l'anthropologue n'est compétent qu'à la marge. Le risque de dévoiement de la dimension scientifique de l'anthropologie au profit d'une approche « appliquée » du social sans portée analytique ni critique [10] [30], inhérent à certaines formes de sollicitation, soulève des questions à la communauté des chercheurs sur les usages sociaux de leur discipline - ou du terme qui la désigne. Enfin, le statut d'anthropologues «embarqués » - c'est-à-dire engagés dans la riposte ${ }^{10}$ - comporte le risque de perte de leur nécessaire distance critique et pousse à produire des avis prescriptifs alors que les études ethnographiques sont plus légitimes pour fournir des orientations ou des explications que des «modes d'emploi ».

De plus, si la contribution des anthropologues était très attendue à propos des perceptions des populations ou des obstacles culturels locaux à l'efficacité des interventions sanitaires, ils ont bien davantage à proposer, comme l'attestent les thèmes de recherche évoqués dans cette esquisse de bilan, notamment pour comprendre les logiques et pratiques de l'ensemble des acteurs. Ils se sont organisés pour aborder «le social » dans un sens « élargi » du terme face aux épidémies émergentes [32], notamment en créant des espaces d'échanges au niveau de la sous-région de l'Afrique de l'ouest du centre. Ainsi, le Réseau ouest africain Anthropologie des Épidémies Émergentes est en mesure d'investiguer des phénomènes sociaux significatifs en

${ }^{10}$ Ce terme a d'abord été utilisé dans les débats critiques sur l'engagement d'anthropologues dans des unités de l'armée américaine pour comprendre les cultures locales en Afghanistan et en Irak [31]. amont de la diffusion du virus et en aval de la flambée épidémique dans des pays à risque épidémique comme dans les pays touchés [33]. La présence d'anthropologues membres du réseau dans 10 pays permet d'aborder les questions à une échelle pertinente sur le plan épidémiologique au vu de la circulation des populations dans des contextes structurels communs. Le réseau offre ainsi les conditions méthodologiques pour mener une analyse anthropologique en développant une approche comparative qui fait apparaître les faits saillants et différences, distinctions ou inégalités ${ }^{11}$. Cette réponse d'anthropologues au-delà de la crise est en phase avec la perspective de "préparation » (preparedness) à d'éventuelles réemergences d'Ebola, prioritaire dans les projets de création de centres africains de contrôle et prévention des maladies, comme dans les outils de mobilisation de la recherche ${ }^{12}$.

\section{Conclusion}

Au vu de l'expérience de l'épidémie d'Ebola, de nombreux acteurs considèrent désormais que les recherches ou les interventions en santé publique face aux épidémies doivent systématiquement impliquer des anthropologues. Il reste à examiner quelle place sera accordée à des analyses anthropologiques approfondies dépassant le temps de la crise sanitaire et s'intéressant aux pays à risque, et si les initiatives collectives d'anthropologues organisés en réseaux seront soutenues par l'attribution de ressources indispensables face aux épidémies émergentes ou ré-émergentes en cours en Afrique de l'Ouest (Zika, Lassa, dengue, fièvre jaune, etc.).

Aucun conflit d'intérêt déclaré

\section{Références}

1. OMS. Flambées Épidémiques de Maladie À Virus Ebola et Marburg : Préparation, Alerte, Lutte et Évaluation. Genève : OMS ; 2014. http://who.int/csr/disease/ebola/manual_EVD/fr/.

${ }^{11}$ Le Réseau ouest africain Anthropologie des Epidémies Emergentes, administré par l'IRD (UMI TransVIHMI) a reçu le soutien d'Expertise France de 2015 à mi-2016.

${ }^{12}$ Notamment le réseau REACTing (AVIESAN, INSERM) qui a soutenu les recherches menées en 2014-15. 
2. Olivier de Sardan JP. La rigueur du qualitatif : Les contraintes empiriques de l'interprétation socio-anthropologique. Éditions Academia, 2008

3. Hewlett BS, Amola RP. Cultural Contexts of Ebola in Northern Uganda. Emerging Infectious Diseases 2003;9(10):1242-48. doi:10.3201/eid0910.020493.

4. Hewlett BS. Ebola, Culture and Politics: The Anthropology of an Emerging Disease. Belmont, CA: 2007.

5. Epelboin A. L'anthropologue dans la réponse aux épidémies: science, savoir-faire ou placebo? Bulletin Amades. Anthropologie Médicale Appliquée au Développement Et à la Santé, 2009;78. http://amades.revues.org/1060.

6. Epelboin A. Ebola, ce n'est pas une maladie pour rire, Film $56 \mathrm{mn}$, 2007, https://www.canal-u.tv/video/smm/ebola_ce_n_est_ pas_une_maladie_pour_rire.13710

7. Epelboin A. Ebola En Guinée Conakry: Hôpital Sécurisé Par MSF, Guéckédou, Avril 2014. Film 31 mn, 2014. http://www.canal-u.tv/ video/smm/ebola_en_guinee_conakry_hopital_securise_par_ msf_gueckedou_avril_2014.14673.

8. The Lancet. Ebola in West Africa: Gaining Community Trust and Confidence. Éditorial. The Lancet 2014;383(9933):1946. doi:10.1016/S0140-6736(14)60938-7.

9. SciDevNet. Ebola: Les anthropologues, composante clé de la riposte. SciDev.Net Afrique Sub-Saharienne. 10 août 2014. http://www. scidev.net/index.cfm?originalUrl=afrique-sub-saharienne/sante/ article-de-fond/ebola-les-anthropologues-composante-cl-de-lariposte.html.

10. Faye SL. L'، exceptionnalité " d'Ebola et les " réticences " populaires en Guinée-Conakry. Réflexions à partir d'une approche d'anthropologie symétrique. Anthropologie \& Santé. 2015;11. doi:10.4000/anthropologiesante.1796.

11. Anoko JN. Communication with Rebellious Communities during an Outbreak of Ebola Virus Disease in Guinea: An Anthropological Approach. Ebola Response Anthropology Platform. 2014. http:// www.ebola-anthropology.net/case_studies/ communication-with-rebellious-communities-during-an-outbreak-of-ebola-virus-disease-in-guinea-an-anthropologicalapproach/.

12. Faye SL. Épidémie d'Ebola en Guinée Conakry: L'anthropologie dans l'urgence sanitaire. La santé en action. Revue de I'INPES, 2015a; 432:37-40.

13. Migliani R, Keita S, Diallo B, Mesfin S, Perea W, Dahl B et al. Aspects épidémiologiques de la maladie à virus Ebola en Guinée (décembre 2013-avril 2016). Bulletin de la Société de pathologie exotique, 2016. doi:10.1007/s13149-016-0511-4.

14. Leach M. Time to Put Ebola in Context. Bulletin of the World Health Organization, 2010;88(7):488-89. doi:10.2471/BLT.10.030710.

15. Niang $\mathrm{Cl}$. Ebola : une épidémie postcoloniale. Politique étrangère, 2014;(4):97-109. doi:10.3917/pe.144.0097.

16. Wilkinson A, Leach M. Briefing: Ebola-myths, Realities, and Structural Violence. African Affairs 2015;114(454):13648.

17. Lachenal G. Outbreak of Unknown Origin in the Tripoint Zone. Limn, 2014;5. http://limn.it/outbreak-of-unknown-origin-in-thetripoint-zone/.

18. Richardson ET, Barrie MB, Kelly JD, Dibba Y, Koedoyoma S, Farmer PE. Biosocial Approaches to the 2013-2016 Ebola Pandemic. Health and Human Rights Journal, 2015;18(1). https://www.hhrjournal. org/2015/12/biosocial-approaches-to-the-2013-2016ebola-pandemic/.

19. Fairhead J. Understanding Social Resistance to Ebola Response in the Forest Region of the Republic of Guinea: An Anthropological Perspective. 2016. African Studies Review. À paraître

20. Fribault M. Ebola en Guinée : violences historiques et régimes de doute. Anthropologie \& Santé, 2015;11. doi:10.4000/ anthropologiesante.1761.

21. ACAPS. Guinea: Resistance to the Ebola Response, Thematic Note. 2015. April 24.

22. ERAP. Stigma and Ebola: An Anthropological Approach to Understanding and Adressing Stigma Operationally in the Ebola Response. Policy Brienfing Note. Ebola Response Anthropology Platform. 2014. http://www.ebola-anthropology.net/key_ messages/stigma-and-ebola-an-anthropological-approach-tounderstanding-and-addressing-stigma-operationally-in-the-ebolaresponse/.

23. ERAP. Ebola Survivors: Using a Stepwise Re-Integration Process to Establish Social Contracts between Survivors and Their Home Communities. Ebola Response Anthropology Platform. 2014. http://www.ebola-anthropology.net/key_messages/ ebola-survivors-using-a-stepwise-re-integration-process-to-establishsocial-contracts-between-survivors-and-their-home-communities/.

24. Sow S, Desclaux A, Taverne B et le Postebogui study group. Ebola en Guinée. Formes de la stigmatisation des acteurs de santé survivants. Bulletin Soc Path Exo, 2016;109(4):309-313; doi:10.1007/ s13149-016-0510-5

25. Kleinman A. Four Social Theories for Global Health. The Lancet 2010; 375(9725):1518-19. doi:10.1016/S0140-6736(10)60646-0.

26. Hofman M, Sokhieng A. Introduction. In: The Politics of Fear: MSF and the West African Ebola Epidemic., Oxford University Press. Hofman M, Au S. (eds), 2017:XV-XXIX.

27. Le Marcis F. "Traiter les corps comme des fagots ». Production sociale de l'indifférence en contexte Ebola (Guinée). Anthropologie \& Santé, 2015;11. http://anthropologiesante.revues.org/1907.

28. Desclaux A, Ndione AG, Badji D, Sow K. La surveillance des personnes contacts pour Ébola : effets sociaux et enjeux éthiques au Sénégal. Bulletin de la Société de pathologie exotique, 2016;109 (4):296-302; doi:10.1007/s13149-016-0477-2

29. Abramowitz S. Ten Things That Anthropologists Can Do to Fight the West African Ebola Epidemic. Somatosphere. 2014, September 26. http://somatosphere.net/2014/09/ten-things-that-anthropologists-can-do-to-fight-the-west-african-ebola-epidemic.html.

30. Desclaux A, Sow K. Des anthropologues face à l'épidémie d'Ebola. Journal des anthropologues. 2016;144-145:263-69.

31. Bonhomme J. Anthropologues Embarqués. La Vie Des Idées. 2007, December 4. http://www.laviedesidees.fr/Anthropologuesembarques.html.

32. Brown $H$, Kelly AH, Marí Sáez A, Fichet-Calvet E, Ansumana $R$, Bonwitt J, et al. Extending the "Social": Anthropological Contributions to the Study of Viral Haemorrhagic Fevers. PLoS Neglected Tropical Diseases, 2015;9(4). doi:10.1371/journal. pntd.0003651.

33. Egrot $\mathrm{M}$, et Comité de coordination du RAEE. Ebola et les sciences sociales. Expériences à partir du Réseau ouest africain Anthropologie des Maladies Émergentes. Transition Humanitaire et Réflexion Éthique En Côte d'Ivoire. 2017, sous presse. 